\title{
Application of an Evolutionary Optimization Technique to Routing in Mobile Wireless Networks
}

\author{
Ibraheem K. Ibraheem, Ph.D \\ Department of Electrical Engineering \\ College of Engineering / Baghdad University
}

\author{
Alyaa A. Alhussainy \\ Department of Electrical Engineering \\ College of Engineering / Baghdad University
}

\begin{abstract}
The main goal of this work is to consider the problem of path optimization between the sender and receiver in a dynamic mobile network. A new adaptive algorithm based on Evolutionary technique called Genetic Algorithm (AGA) has been proposed to find out the Optimal Path in dynamic nature problem. In this paper, the proposed AGA for solving the shortest path routing problem is presented to find the shortest path in a mobile wireless networks to satisfy the minimum end-to-end delay quality of service $(Q o S)$. The proposed algorithm provides the solution of optimal path using a technique that is inspired by the natural process that is initial population, selection crossover and mutation. The proposed AGA uses a multiple selection methods to improve the performance of particular implementations. Simulations have been done using both MATLAB and Visual basic environments and the results show that the proposed algorithm performs excellently by finding a path with minimum end-to-end delay between source and destination; it finds the shortest path in dynamic environment efficiently and quickly adapts to the environmental changes (i.e., the network topology change) and produces good solutions after each change.
\end{abstract}

\section{General Terms}

Computer networks, Data routing, Genetic Algorithm, shortest path, wireless networks.

\section{Keywords}

Quality of Service $(Q o S)$, mobile networks, end-to-end delay, network traffic, networks nodes, evolutionary optimization.

\section{INTRODUCTION}

In recent years, mobile networks have spread widely. So, the conventional techniques of connecting networks failed to satisfy the requirements imposed by our new way of living. The wired networking restrict the movement of the users dramatically. Wireless networks, however, remove such restrictions and allow a great deal more of free mobility of the network user. As a result, wireless technologies are invading the traditional realm of fixed networks [1]. Data network routing algorithm is transferring packets from source node to destination node. Simple routing algorithms that determine forwarding paths based on the minimum number of hops or end-to-end delay to a specified destination are no longer adequate. This demonstrates the necessity for powerful dynamic traffic routing that aims at improving the utilization of a transportation network capacity, which is a thoughtprovoking task that involves solving a multifaceted optimization problem. Routing in general involves two entities, that is the routing protocol and the routing algorithm. The later manages the dynamics of the routing process, i.e. taking the state of the network and its available network resources and allotting this information throughout the network, while the routing algorithm uses this information to compute the paths that enhances a criterion and/or follow constraints. Routing strategies can be classified according to Mechanisms for activating search for feasible routes into [2]: Pro-active routing, In this approach, routes various destinations are preserved at all times (pre-computed), whether they are required or not, while in Reactive routing, routes to destinations are computed when they are needed (ondemand). This approach reduces latency, on the account of slower response times. A routing technique sometimes applied in the Internet in source routing, which is a technique in which the source specifies the whole path to the destination in the packet header [2]. There are several search algorithms for the shortest path (SP) problem: the breadth-first search algorithm, the Dijkstra algorithm and the Bellman-Ford algorithm [3]. Since these algorithms are effective in fixed infrastructure wireless or wired networks, but they exhibit a high computational complexity for real-time communications involving swiftly changing network topologies. In MANET nodes are free to move randomly, thus the network topology which is typically multi-hop may change randomly in unpredictable time. Since the network topologies are changing, it is necessary to change the routes randomly and discover the best path in real time. Genetic algorithms are an optimization technique that deals with the uncertainties related with changing environment [4]. Researchers have resorted to a wide variety of techniques. Leonardo Badia et al. [5] proposed and used a technique based on Genetic Algorithms (GAs). The technique is suitable for this purpose as it provides a good trade-off between fast computation and the overall goodness of the solution found. GAs would seem to be quite promising for solving more complex WMN models than the one dealt with in this paper, such as those including multiple flows and multi-radio multi-channels. Hui Cheng et al. [6], a multi-population GAs with immigrant's scheme was proposed to solve the dynamic shortest path (SP) problem in MANETs which is the representative of new generation wireless networks. The experimental results show that the proposed GAs can quickly adapt to the environmental changes (i.e., the network topology change) and produce good solutions after each change. While authors in [7] examined two aspects concerning the influence of unidirectional, they evaluated the benefit from utilizing unidirectional links for routing, as opposed to using only bidirectional links. Our evaluations are based on three transmit power assignment models that reflect some realistic network scenarios with unidirectional links links on routing performance in multihop wireless networks. T. G. Nair et al. [8] discussed the implementation of GA in the SP routing problem in MANET. The implementation uses GAs with immigrants and memory schemes to solve the dynamic SP routing problem (DSPRP) in MANETS. Once the network topology changes, GA with new immigrants shows better convergence result than GA with memory scheme. A new routing algorithm for WMN, namely 
Adaptive Simulated Annealing, based on Ant Colony Optimization algorithm(ACO) and simulated annealing algorithm(SA) was presented in [9]. This algorithm not only combined the adaptation, robustness and distributed, decentralized merit of ACO but also used simulated annealing to remedy the weakness of ant algorithm and increase the convergence rate of ACO. Halil Yetgin et al [10] invoked the Non-dominated Sorting based Genetic Algorithm-II (NSGAII) and the Multi Objective Differential Evolution (MODE) algorithm for finding optimal routes from a given source to a given destination in the face of conflicting design objectives, such as the dissipated energy and the end-to-end delay in a fully-connected arbitrary multi-hop network. In [11] researchers presented the Mesh Routing Algorithm (MRA), a dynamic programming approach to compute high-capacity paths while simultaneously bounding the end-to-end delay. The proposed algorithm also provides the option of routing through multiple link-disjoint paths, such that the amount of traffic through each path is proportional to its capacity. In [12] a new method based on the combination of the ant algorithm and local search algorithm to find shortage path in wireless networks. According to the results of combined algorithms, it significantly optimizes the algorithm for finding the shortest route. Mala C.et al. [13], Genetic Algorithm has been used to determine the optimal multicast routing satisfying the quality of service requirements such as total delay, total delay jitter, packet loss and total bandwidth. New parameters Total Buffer Size and Queuing Delay added along with the other parameters and the simulation results through MATLAB suggest that convergence is faster with better results. The remainder of the paper is organized as follows: section 2 explains the genetic operators used in algorithm, while the developed genetic algorithm for routing discovery in dynamic network proposed in section 3. In section 4 the implementation details are given. Simulation results presented and discussed in Section 5. The paper is concluding in Section 6.

\section{GENETIC ALGORITHM (GA)}

GA are an evolutionary optimization approach, they are particularly applicable to problems which are large, non-linear $\&$ possibly discrete in nature. GA try to work on principle of natural selection, as in natural selection over the time individuals with "good" genes survive whereas "bad" ones are rejected. GA collects the possible alternative solutions of a problem as a genetic string. Simple GA uses three operators with probabilistic rules [14]: 1) reproduction, 2) crossover and 3 ) mutation, as shown in Fig. 1. Firstly selective reproduction is applied to the current population so that the string makes a number of copies proportional to their own fitness. This results in an intermediate population. Secondly, GA select "parents" from the current population with a bias that better chromosome are likely to be selected. Thirdly, GA reproduces "children" (new strings) from selected parents using crossover and/or mutation operators. Crossover is basically consists in a random exchange of bits between two strings of the intermediate population. Finally, the mutation operator alters randomly some bits of the new strings. This algorithm terminates when an acceptable solution is found or when a predetermined limited number of iteration is reached [14, 15].

The application of GA on routing in mobile wireless networks can be described as follows, GA maintains a population of candidate solutions (genetic string), where each candidate solution called a chromosome. The chromosome consists of sequences of positive integers that represent the IDs of nodes through which a routing path passes. Each locus of the chromosome represents an order of a node in a routing path. The gene of first locus always reserved for the source node. The length of the chromosome is variable, but it should not exceed the maximum length, which is the total number of nodes in the network [14], see Fig. 2 [16]. A chromosome (routing path) encodes the problem by listing up node IDs from its source node to its destination node based on topological information database (routing table) of the network. A set of chromosomes forms a population, which is evaluated and ranked by fitness evaluation function. The fitness evaluation function plays a critical role in GA because it provides information how good is each candidate. The crossover operator exchanges sub-routes between two chromosomes. Firstly, select the chromosomes according the probability of crossover operator. Secondly, apply crossover to the selected chromosomes using the path crossover operator by selecting randomly two paths that should have the same source and destination nodes. A node is randomly selected as a crossing site from the possible crossing sites and exchanges sub-routes. Figure 3 shows the crossover operator on a pair of paths P1 and P2 from node 1 to node 4 . Their potential crossing sites is node 3 . When we select node 3 as a crossing site, the new offspring are generated by exchanging the subroutes [17].

The path mutation operator is important to avoid loops in a route, and avoiding local minimum in the solution. It generates an alternative path from a chromosome. To perform a mutation, first, a node is randomly selected from the path, which is called a mutation node. Then another node is randomly selected from the nodes directly connected to the mutation node. Figure 4 shows an example of a mutation operator. First, suppose that node 7 is selected as a mutation node. Second, node 8 is randomly selected from the neighbors of the mutation node. Third, generate random path from node 0 to node $8(\mathrm{r} 1)$, and random path between node 8 to node 15 $(\mathrm{r} 2)$. We finish the mutation by connecting the sub-routes $\mathrm{r} 1$ and $\mathrm{r} 2$. An offspring $\mathrm{V}$ ' isn't generated if any duplication of nodes exists in sub-routes $\mathrm{r} 1$ and $\mathrm{r} 2$. This is because we need to avoid creating any loop in a route. 


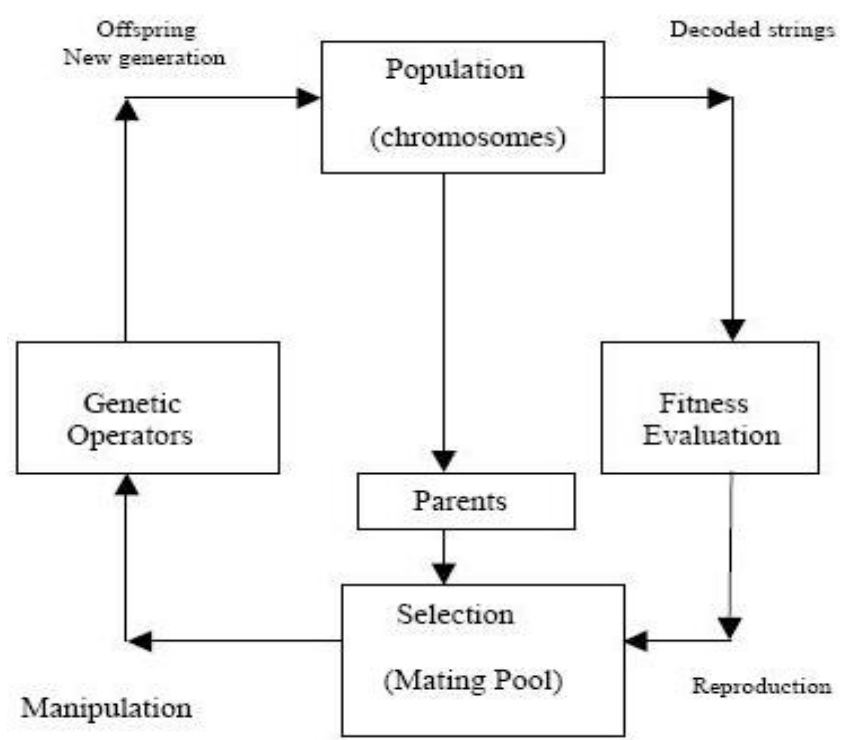

Fig. 1: Genetic Algorithm Stages.

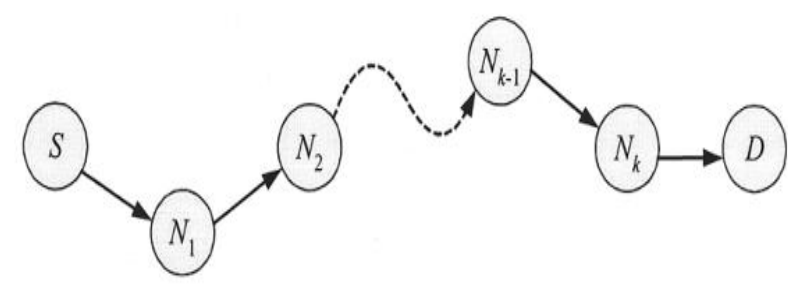

\begin{tabular}{rl|l|l|l|l|l|l|}
\multicolumn{1}{c}{ locus : } & 1 & 2 & \multicolumn{1}{c}{3} & \multicolumn{1}{c}{$l-2$} & $l-1$ & $l$ \\
\cline { 2 - 8 } chromosome : & $S$ & $N_{1}$ & $N_{2}$ & $\ldots$ & $N_{k-1}$ & $N_{k}$ & $D$ \\
\hline
\end{tabular}

Fig. 2: Example of routing path and encoding scheme[16].

\begin{tabular}{|l|l|l|l|l|l|l|l|l|l|l|l|}
\hline Path P1 & 0 & 2 & 3 & 7 & 9 & 11 & 12 & 15 & 17 & 18 & 20 \\
\hline Path P2 & 0 & 4 & 5 & 7 & 10 & 11 & 12 & 15 & 16 & 20 & \multicolumn{1}{c|}{} \\
selected point \\
\begin{tabular}{|l|l|l|l|l|l|l|l|l|l|l|l|}
\hline Child P1' & 0 & 2 & 3 & 7 & 9 & 11 & 12 & 15 & 16 & 20 & \\
\hline Child P2' & 0 & 4 & 5 & 7 & 10 & 11 & 12 & 15 & 17 & 18 & 20 \\
\hline
\end{tabular}
\end{tabular}

Figure 3: Example of single point crossover [17].

When a common node in a pair of chromosomes does not exist, a crossing site cannot be selected; therefore, it is impossible to perform the crossover operator.

\begin{tabular}{|l|l|l|l|l|l|l|l|l|}
\hline Parent V & 0 & 3 & 5 & 6 & 7 & 10 & 12 & 15 \\
\hline
\end{tabular}
\begin{tabular}{|l|l|l|l|l|}
\hline Sub route r1 & 0 & 2 & 4 & 8 \\
\hline
\end{tabular}

\begin{tabular}{|l|l|l|l|l|l|l|l|l|}
\cline { 2 - 6 } \multicolumn{10}{c|}{} & Sub route 2 & 8 & 10 & 12 & 15 \\
\hline Offspring V' & 0 & 2 & 4 & 8 & 10 & 12 & 15 \\
\hline
\end{tabular}

Fig. 4: Example of Mutation.

\subsection{Selection Methods}

The selection mechanism determines which individuals are chosen for mating (reproduction) and how many offspring each selected individual produces. The main principle of selection strategy is "the better is an individual; the higher is its chance of being parent."[18]. There is many types of selection methods, the selection methods used in this paper are:

1-Roulette Wheel selection (RWS): In roulette wheel, individuals are selected with a probability that is directly proportional to their fitness values i.e. an individual's selection corresponds to a portion of a roulette wheel. The probabilities of selecting a parent can be seen as spinning a roulette wheel with the size of the segment for each parent being proportional to its fitness. Obviously, those with the largest fitness (i.e. largest segment sizes) have more probability of being chosen. The fittest individual occupies the largest segment, whereas the least fit have correspondingly smaller segment within the roulette wheel. The circumference of the roulette wheel is the sum of all fitness values of the individuals [19].

2- Tournament selection (TS): Tournament selection involves running several "tournaments" among a few individuals chosen at random from the population. The winner of each tournament (the one with the best fitness) is selected for crossover. Selection pressure is easily adjusted by changing the tournament size. If the tournament size is larger, weak individuals have a smaller chance to be selected, see Fig. 3. The number of individuals competing in each tournament is referred to as tournament size, commonly set to 2 (also called binary tournament) [19].

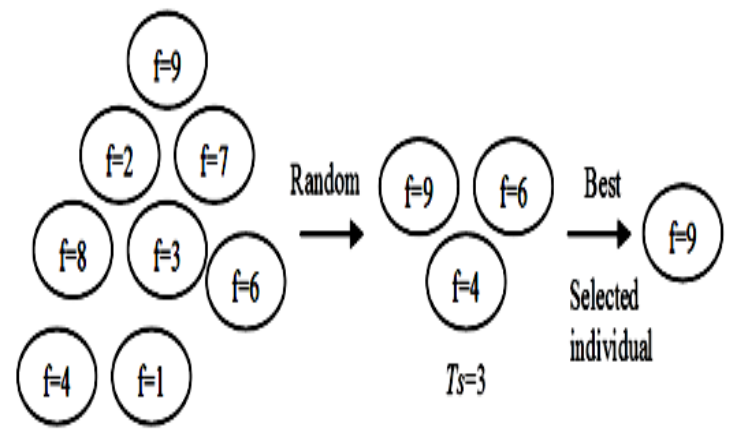

Fig. 5: Selection strategy with tournament mechanism [19]. 
3- Steady state selection (SSS): It differs from the generic GA in that tournament selection does not replace the selected individuals in the population, and instead of adding the children of the selected parents into the next generation, the two best individuals out of the two parents and two children are added back into the population so that the population size remains constant.

$$
\text { [18]. }
$$

4-Boltizmann selection (BS): a continuously varying "temperature" controls the rate of selection according to a present schedule. The temperature starts out high, which means that selection pressure is low and vice versa thereby allowing GA to narrow in ever more closely to the best part of the search space[20, 21].

5-Sigma scaling selection (SigSS): keeps the selection pressure relatively constant over the course of the run rather than depending on the fitness variances in the population. Under sigma scaling, an individual's expected value is a function of its fitness, the population mean, and the population standard deviation [20].

6-Rank selection (RS): Rank selection first ranks the population and then every chromosome receives fitness from this ranking. The worst will have fitness 1 , second worst 2 etc. and the best will have fitness $N$ (number of chromosomes in population). After this all the chromosomes have a chance to be selected. But, this method can lead to slower convergence, because the best chromosomes do not differ so much from other ones [19].

\section{A PROPOSED AGA FOR ROUTING IN MOBILE WIRELESS NETWORKS}

A mobile wireless network under consideration is represented as a connected graph with $N$ nodes. The metric of optimization is the end-to-end delay of the path between the nodes. The total end-to-end delay is the sum of delays of the individual hops. The goal is to find the path with minimum total end-to-end delay between the source and destination nodes. This part presents a simple and effective proposed Adaptive genetic algorithm (AGA) to find the shortest path with minimum total end-to-end delay as quality of service $(Q o S)$. The details of the algorithm are given in the following subsections; while the investigation of the performance is achieved via a simulation work in the next section. Our contribution in this work lies in two points: firstly, the proposed algorithm deals with dynamic topology network where nodes are mobile, and the second contribution is the adaptive nature of the reproduction stage for the proposed algorithm. The selection methods used in the proposed AGA are the six selection methods mentioned in the previous section. With the adaptive selection method means, each generation is obtained using a selection method with most suitable for this generation (best fitness). The proposed AGA includes the following steps:

\section{Initial populations}

This initial process is used to compose the routing tables (all chromosomes) in the current generation. Each chromosome includes a random routing table for the given network topology.

2. Evaluating the fitness function Evaluation of fitness value of routes is based on the delay of links between nodes in the route. Each solution in the population will be assigned a value calculated from its total link cost. The fitness function of a chromosome is calculated as a summation of the each node delay to produce path delay, so the objective to minimize the total delay.as follows:

$$
F=\min \left\{\sum \text { Delay }(P(V, E))\right\}
$$

Where $F$ is Fitness function, $P$ is a path between source node and destination node, $V$ set of nodes represent routers, and $E$ set of links that connect the routers.

\section{Selection Methods}

We apply the reproduction operator in our Proposed AGA as follows, with six selection methods explained in section 2 . They are evaluated at the same time and for every generation and for all of the chromosomes, the fitness value of each method is represented by its maximum and minimum values, see Table 2. In the end-to-end delay $Q o S$ measure, our concern is with the minimum values of the fitness values (Minimizing the end-to-end delay). A chromosome with a highest fitness value (minimum of the total end-to-end delay, i.e. eq. (1)) has more chance to be selected using one of the six selection methods that has the minimum fitness value.

\section{Path Crossover Operator}

Apply crossover to the selected chromosomes using the path crossover operator by selecting randomly two paths that should have the same source and destination nodes.

\section{Mutation Operator}

Perform the mutation operator by randomly choosing a mutation node in the path. The rest of the procedure follows Fig. 4.

\section{SIMULATION AND RESULTS}

A mobile wireless Network with 50 nodes has been selected for simulation connection were designed to allow multi path alternate from source to destination. Figure (6) .A mobile wireless network can be expressed as a weighted graph $\mathrm{G}=(V, E)$, where $V$ set of nodes and $E$ set of links. Topology area: Nodes are distributed randomly on $1000 * 1000 \mathrm{~m}^{2}$.

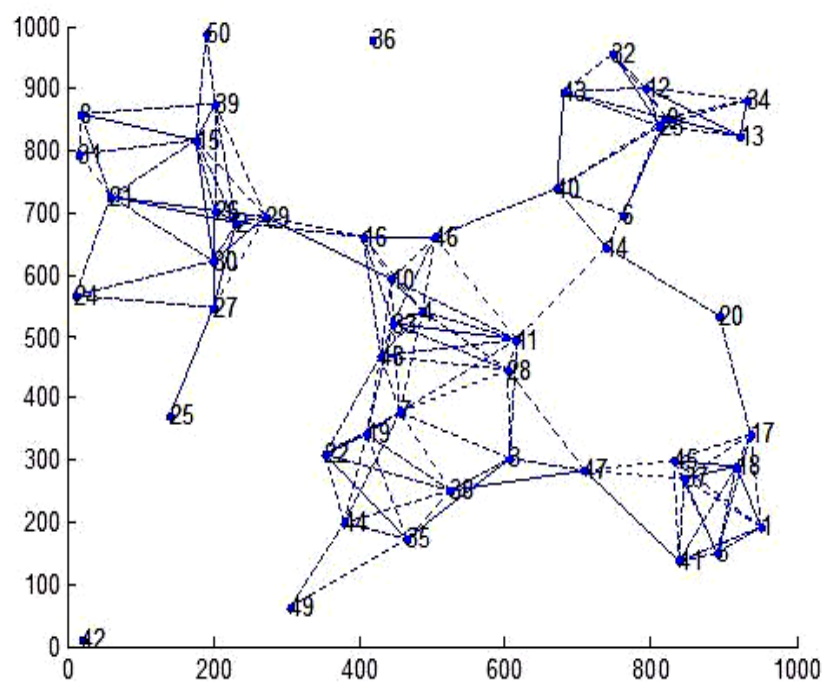

Fig. 6: Network Topology of 50 nodes. 
Table 1: Rrouting Table for mobile wireless networks with end-to-end delay $Q o S$.

\begin{tabular}{|c|c|c|c|c|c|c|c|c|c|c|c|c|}
\hline 总 & \multicolumn{11}{|c|}{ Path } & \multirow{2}{*}{$\begin{array}{l}\begin{array}{c}\text { fitness } \\
\text { delay }\end{array} \\
2.2000\end{array}$} \\
\hline 1 & 1 & 29 & 50 & 0 & 0 & 0 & 0 & 0 & 0 & 0 & 0 & \\
\hline 2 & 1 & 22 & 23 & 50 & 0 & 0 & 0 & 0 & 0 & 0 & 0 & 1.9000 \\
\hline 3 & 1 & 12 & 20 & 48 & 50 & 0 & 0 & 0 & 0 & 0 & 0 & 2.1000 \\
\hline 4 & 1 & 18 & 42 & 32 & 17 & 28 & 50 & 0 & 0 & 0 & 0 & 1.8000 \\
\hline 5 & 1 & 36 & 50 & 0 & 0 & 0 & 0 & 0 & 0 & 0 & 0 & 1.3000 \\
\hline 6 & 1 & 22 & 43 & 3 & 36 & 50 & 0 & 0 & 0 & 0 & 0 & 1.4000 \\
\hline 7 & 1 & 48 & 49 & 33 & 44 & 3 & 28 & 50 & 0 & 0 & 0 & 2.0000 \\
\hline 8 & 1 & 16 & 18 & 2 & 50 & 0 & 0 & 0 & 0 & 0 & 0 & 1.9000 \\
\hline 9 & 1 & 12 & 43 & 17 & 50 & 0 & 0 & 0 & 0 & 0 & 0 & 2.0000 \\
\hline 10 & 1 & 5 & 7 & 46 & 14 & 50 & 0 & 0 & 0 & 0 & 0 & 1.5000 \\
\hline 11 & 1 & 17 & 4 & 50 & 0 & 0 & 0 & 0 & 0 & 0 & 0 & 1.3000 \\
\hline 12 & 1 & 12 & 41 & 16 & 4 & 50 & 0 & 0 & 0 & 0 & 0 & 1.3000 \\
\hline 13 & 1 & 21 & 7 & 8 & 42 & 29 & 28 & 50 & 0 & 0 & 0 & 1.8000 \\
\hline 14 & 1 & 32 & 30 & 36 & 15 & 50 & 0 & 0 & 0 & 0 & 0 & 2.3000 \\
\hline 15 & 1 & 47 & 49 & 31 & 50 & 0 & 0 & 0 & 0 & 0 & 0 & 2.2000 \\
\hline 16 & 1 & 19 & 50 & 0 & 0 & 0 & 0 & 0 & 0 & 0 & 0 & 2.0000 \\
\hline 17 & 1 & 6 & 35 & 30 & 50 & 0 & 0 & 0 & 0 & 0 & 0 & 1.8000 \\
\hline 18 & 1 & 6 & 43 & 50 & 0 & 0 & 0 & 0 & 0 & 0 & 0 & 1.2000 \\
\hline 19 & 1 & 32 & 50 & 0 & 0 & 0 & 0 & 0 & 0 & 0 & 0 & 1.4000 \\
\hline 20 & 1 & 8 & 16 & 28 & 50 & 0 & 0 & 0 & 0 & 0 & 0 & 1.7000 \\
\hline 21 & 1 & 24 & 31 & 23 & 42 & 50 & 0 & 0 & 0 & 0 & 0 & 1.8000 \\
\hline 22 & 1 & 15 & 32 & 50 & 0 & 0 & 0 & 0 & 0 & 0 & 0 & 2.4000 \\
\hline 23 & 1 & 23 & 39 & 18 & 34 & 50 & 0 & 0 & 0 & 0 & 0 & 1.7000 \\
\hline 24 & 1 & 17 & 50 & 0 & 0 & 0 & 0 & 0 & 0 & 0 & 0 & 1.7000 \\
\hline 25 & 1 & 2 & 31 & 27 & 50 & 0 & 0 & 0 & 0 & 0 & 0 & 1.3000 \\
\hline
\end{tabular}

The initializations for our proposed algorithm are: source node $=1$, destination node $=50$, initial population $=50$, pop size $=50$, Crossover probability $=0.75$, Mutation probability $=0.01$, number of generation $=100$. Table 1 shows the inial population of 50 routes with their delays, whereas the fitness value of each selection method is represented by its maximum and minimum fitness values for the $1^{\text {st }}$ generation in Table 2.

Discussion: There are 50 possible paths from 1 to 50 as initial populations. The list is shown in Table 1 . Table 2 illustrate the six different selection criteria used in this simulation, where all of them are evaluated at the same time for each generation in proposed AGA, the minimum and maximum values of the fitness function are evaluated using these criteria, the best selection method is picked for that generation which has the minimum fitness value which is the case of casestudy, because our $Q o S$ is the optimization of the path that has minimum end-to-end delay. The simulation is terminated when the number of generations reached for optimal solution is 100 .

\section{Table 1: Continued}

\begin{tabular}{|c|c|c|c|c|c|c|c|c|c|c|c|c|}
\hline $\mathbf{2 6}$ & $\mathbf{1}$ & $\mathbf{3 4}$ & $\mathbf{2 6}$ & $\mathbf{3 5}$ & $\mathbf{3}$ & $\mathbf{1 3}$ & $\mathbf{5 0}$ & $\mathbf{0}$ & $\mathbf{0}$ & $\mathbf{0}$ & $\mathbf{0}$ & $\mathbf{1 . 6 0 0 0}$ \\
\hline 27 & 1 & 28 & 11 & 13 & 36 & 22 & 50 & 0 & 0 & 0 & 0 & 2.0000 \\
\hline 28 & 1 & 33 & 50 & 0 & 0 & 0 & 0 & 0 & 0 & 0 & 0 & 1.5000 \\
\hline 29 & 1 & 15 & 43 & 50 & 0 & 0 & 0 & 0 & 0 & 0 & 0 & 1.8000 \\
\hline 30 & 1 & 13 & 2 & 48 & 50 & 0 & 0 & 0 & 0 & 0 & 0 & 1.9000 \\
\hline 31 & 1 & 8 & 17 & 25 & 50 & 0 & 0 & 0 & 0 & 0 & 0 & 1.8000 \\
\hline 32 & 1 & 6 & 50 & 0 & 0 & 0 & 0 & 0 & 0 & 0 & 0 & 1.6000 \\
\hline 33 & 1 & 27 & 48 & 16 & 50 & 0 & 0 & 0 & 0 & 0 & 0 & 1.5000 \\
\hline 34 & 1 & 14 & 45 & 9 & 24 & 50 & 0 & 0 & 0 & 0 & 0 & 1.8000 \\
\hline 45 & 1 & 26 & 15 & 40 & 38 & 24 & 50 & 0 & 0 & 0 & 0 & 1.9000 \\
\hline 36 & 1 & 29 & 47 & 50 & 0 & 0 & 0 & 0 & 0 & 0 & 0 & 1.7000 \\
\hline 37 & 1 & 32 & 41 & 50 & 0 & 0 & 0 & 0 & 0 & 0 & 0 & 1.5000 \\
\hline 38 & 1 & 9 & 38 & 15 & 50 & 0 & 0 & 0 & 0 & 0 & 0 & 1.2000 \\
\hline 39 & 1 & 24 & 33 & 5 & 15 & 50 & 0 & 0 & 0 & 0 & 0 & 1.9000 \\
\hline 40 & 1 & 15 & 32 & 11 & 42 & 16 & 50 & 0 & 0 & 0 & 0 & 1.5000 \\
\hline 41 & 1 & 29 & 11 & 13 & 50 & 0 & 0 & 0 & 0 & 0 & 0 & 2.1000 \\
\hline 42 & 1 & 3 & 11 & 10 & 47 & 42 & 26 & 22 & 2 & 14 & 50 & 1.6000 \\
\hline 43 & 1 & 6 & 15 & 23 & 50 & 0 & 0 & 0 & 0 & 0 & 0 & 1.5000 \\
\hline 44 & 1 & 22 & 48 & 36 & 31 & 50 & 0 & 0 & 0 & 0 & 0 & 1.6000 \\
\hline 45 & 1 & 16 & 40 & 35 & 50 & 0 & 0 & 0 & 0 & 0 & 0 & 1.5000 \\
\hline 46 & 1 & 28 & 21 & 26 & 50 & 0 & 0 & 0 & 0 & 0 & 0 & 1.6000 \\
\hline 47 & 1 & 39 & 3 & 50 & 0 & 0 & 0 & 0 & 0 & 0 & 0 & 1.4000 \\
\hline 48 & 1 & 27 & 19 & 33 & 28 & 50 & 0 & 0 & 0 & 0 & 0 & 1.7000 \\
\hline 49 & 1 & 44 & 18 & 50 & 0 & 0 & 0 & 0 & 0 & 0 & 0 & 2.1000 \\
\hline 50 & 1 & 16 & 43 & 50 & 0 & 0 & 0 & 0 & 0 & 0 & 0 & 2.4000 \\
\hline
\end{tabular}

The optimal path obtained is shown in figure 7 is $\mathbf{1} \mathbf{7 9 5 0}$ with minimum total delay equal to $10.5954 \mathrm{msec}$ resulted from selection method two, tournament selection (TS), where the network nodes are mobile and figure 8 shows the optimal path for different network topology, since our topology is dynamic and the nodes are mobile, the algorithm adapts itself for that change and obtains the optimal solution whatever the network topology of the network is. Figure 9 shows the value of the fitness versus the population size.

Table 2: Initial fitness values for $\mathbf{1}^{\text {st }}$ generation of end-toend delay $Q o S$ optimization.

\begin{tabular}{|c|c|c|c|}
\hline $\begin{array}{c}\text { Selection } \\
\text { method no. }\end{array}$ & $\begin{array}{c}\text { Selection } \\
\text { method }\end{array}$ & Maximum & Minimum \\
\hline 1 & RWS & 13.5732 & 12.0446 \\
\hline 2 & TS & 13.5732 & 11.7425 \\
\hline 3 & SSS & 13.5732 & 12.0446 \\
\hline 4 & BS & 13.5732 & 12.0446 \\
\hline 5 & Sig SS & 13.5732 & 12.0446 \\
\hline 6 & RS & 13.5732 & 11.352 \\
\hline
\end{tabular}




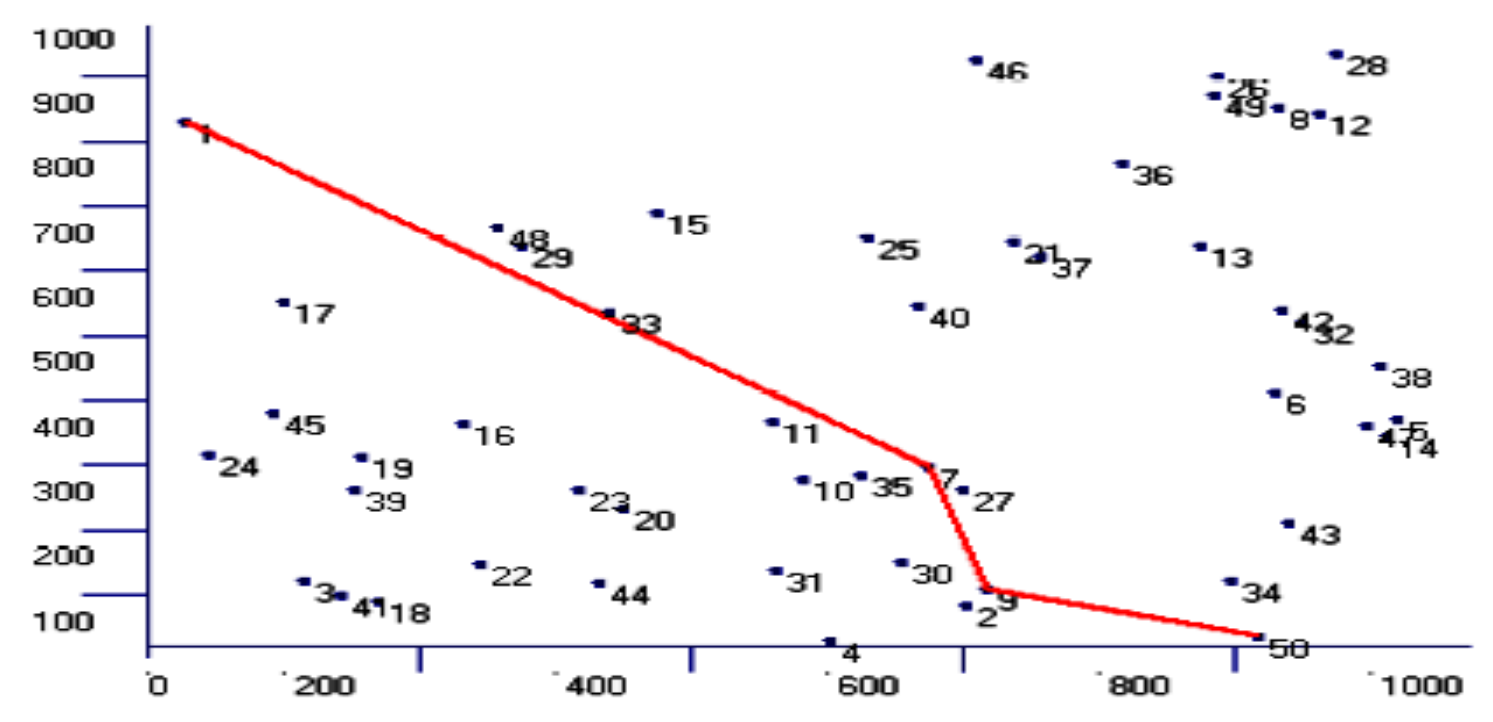

Fig. 7: The shortest path for a network area of $1000 * 1000 \mathrm{~m}^{2}$.

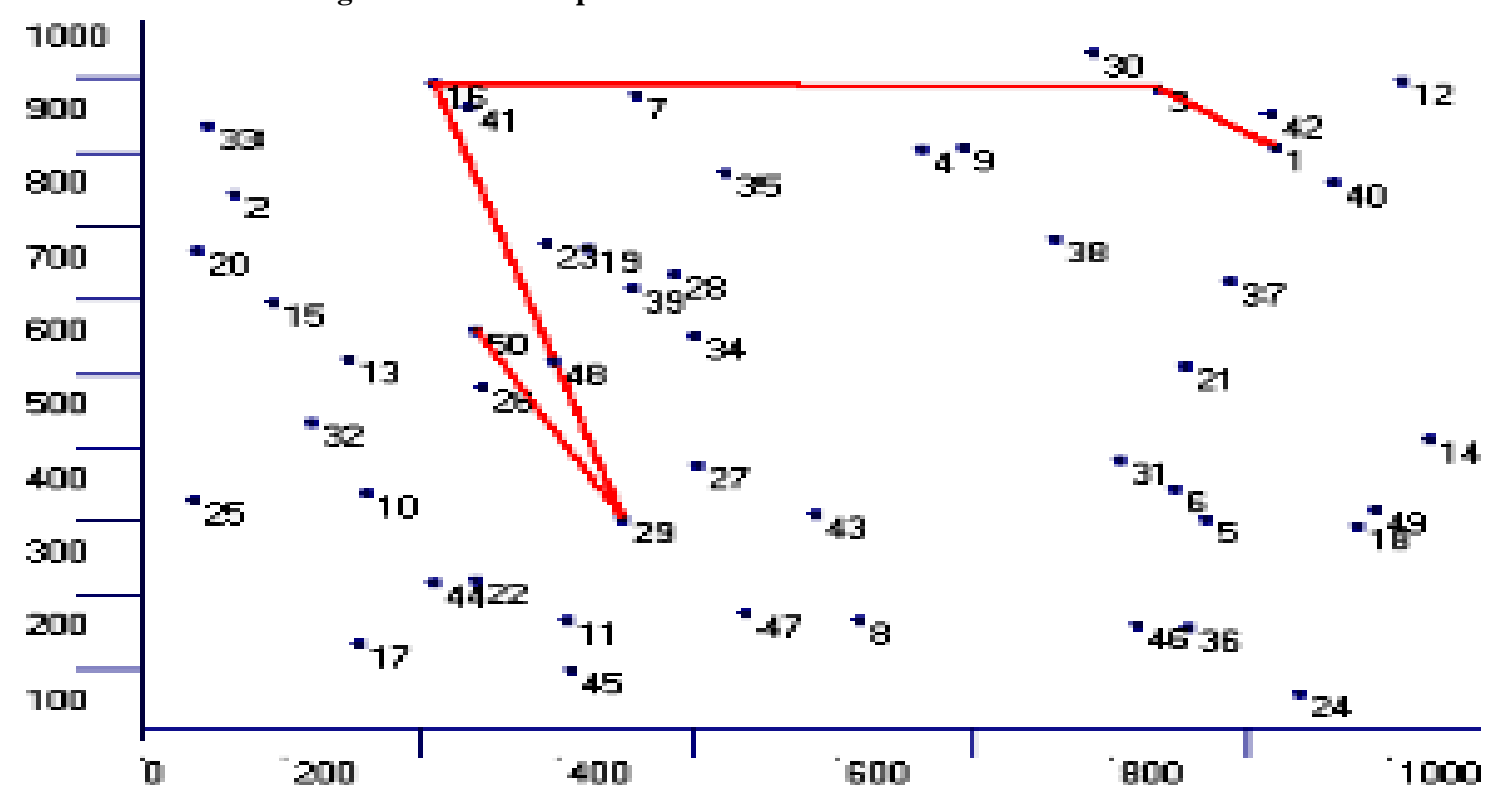

Fig. 8: The shortest path for another network topology. 


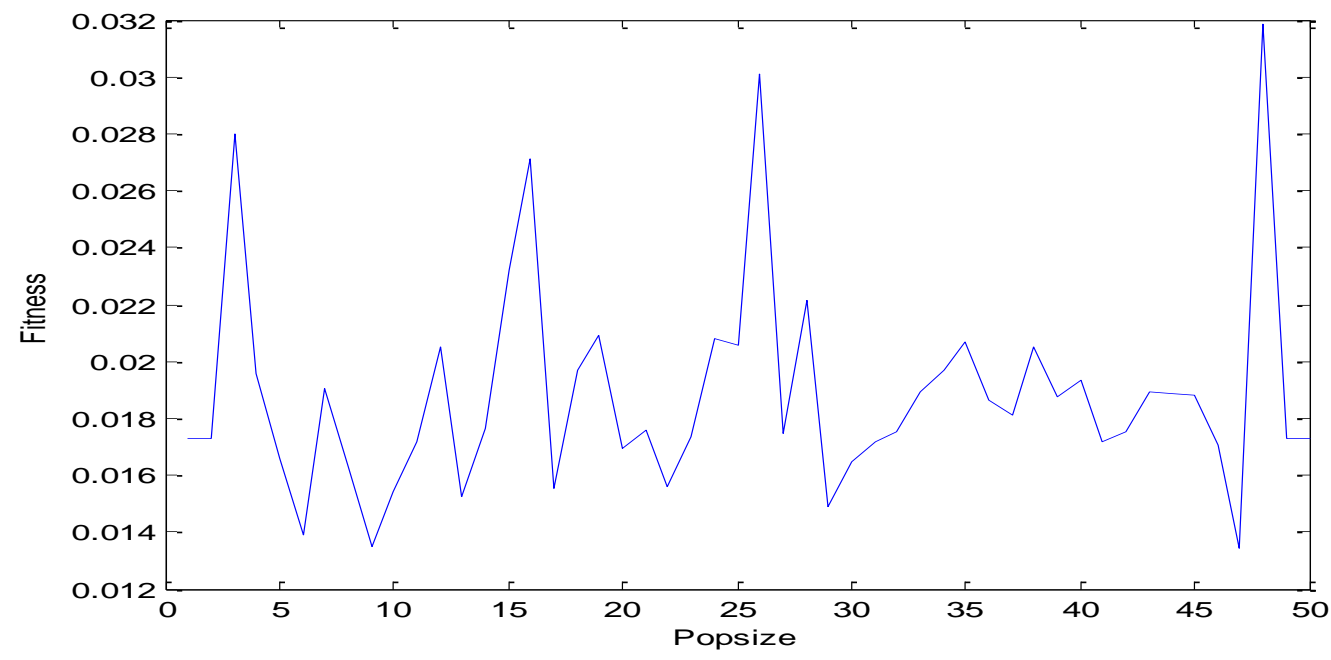

Fig. 9: Fitness and Pop size correlation.

\section{CONCLUSION}

This paper presented adaptive genetic algorithm for solving the shortest path routing problem. The proposed algorithm can search the solution space efficiently and speedily compared with other algorithms through its operators. In our proposed AGA, all the paths of the dynamic network are generated randomly and adaptive genetic operator applied on weighting function to optimize the result or minimize the fitness value for minimum delay as $Q o S$ used in this work. The different selection strategies used in the proposed AGA in this paper significantly affected the performance of the algorithm. Whereas it is widened the search space and improved the selection process. Our work concludes the path, which is obtained as an offspring given the value of best solution (fitness value) which is the delay in our case study. Our proposed AGA performs better and effectively even to changes in the network due to node mobility and topology changes and give optimum path with minimum delay.

\section{REFERENCES}

[1] M. Gast. 2002. 802.11® Wireless Networks: The Definitive Guide. Publisher: O'Reilly, ISBN: 0-59600183-5.

[2] H. N.Saad. 2010. Source routing: Best rout using genetic algorithm. Journal of Kufa for Mathematics and Computer, Vol.1, No.2, Oct., pp.35 - 45 .

[3] R. Kumar and M. Kumar. 2012. Reliable and Efficient Routing Using Adaptive Genetic Algorithm in Packet Switched Networks. IJCSI International Journal of Computer Science Issues, Vol. 9, Issue 1, No 3, January 2012, ISSN (Online): 1694-0814

[4] Z. Ishtar, K. B. Ali. 2013. Optimization of Route in a Network using Genetic Algorithm. International Conference on Advances in Computer Application (ICACA - 2013), Proceedings published in International Journal of Computer Applications ${ }^{\circledR}$ (IJCA) (0975 8887).

[5] L. Badia, A. Botta, L. Lenzini. 2007. Joint Routing and Link Scheduling for Wireless Mesh Networks through Genetic Algorithms. IEEE International Conference 1620 April 2007, Limassol.
[6] H. Cheng, Sh. Yang. 2010. Multi-population Genetic Algorithms with Immigrants Scheme for Dynamic Shortest Path Immigrants Scheme for Dynamic Shortest Path. Lecture notes in computer science, Volume 6024, 2010, pp 562-571.

[7] Mahesh K. Marina, Samir R. Das. 2002. Routing Performance in the Presence of Unidirectional Links in Multihop Wireless Networks. Proceedings of the 3rd ACM international symposium on Mobile ad hoc networking \& computing. ACM, 2002.

[8] T. R. G. Nair, Ms.K. Sooda, Ms.Y.M B. 2011 Enhanced Genetic Algorithm approach for Solving Dynamic Shortest Path Routing Problems using Immigrants and Memory Schemes. International Conference on Frontiers of Computer Science, 7 TH TO 9 TH August 2011, JN Tata Convention Centre, IISc, Bangalore, India.

[9] Y. Zhao, R. Sun, and L. Xu. 2010. An Ant Simulated Annealing Routing Algorithm for Wireless Mesh Network. IEEE International Conference on Internet Technology and Applications. pages 1-4.

[10] H.Yetgin, K. T. K. Cheung and L. Hanzo. 2012 Multiobjective Routing Optimization Using Evolutionary Algorithms. IEEE WIRELESS COMMUNICATIONS AND NETWORKING CONFERENCE, PARIS, APRIL 2012.

[11] J. Crichigno, J. Khoury, M. Y. Wu , and W. Shu. 2008. A Dynamic Programming Approach for Routing in Wireless Mesh Networks. Global Telecommunications Conference, IEEE GLOBECOM.

[12] Fatemeh K. Purian, Reza S. Nodoshan. 2013. A Novel Method to Find the shortest Path in Wireless Networks. Journal of Academic and Applied Studies. Vol. 3, pages $1-14$.

[13] Mala C, A. A. Mahesh, R. Aravind, R. Rajgopal, N. Rajagopalan. B. Nithya. 2011. Simulated Study of QoS Multicast Routing Using Genetic Algorithm. World Applied Programming, Vol (2), Issue (5), May 2012. 342-348, ISSN: 2222-2510,(C 2011 WAP journal. www.waprogramming.com.

[14] R. L. Haupt and S. E. Haupt. 2004. Practical Genetic Algorithm. $2^{\text {nd }}$ Edition, Wiley. 
[15] A. Mohammed., G. Nagib. 2012. Optimal Routing In Ad-Hoc Network Using Genetic Algorithm. Int. J. Advanced Networking and Applications, Volume: 03, Issue: 05, Pages: 1323-1328 (2012).

[16] Ch. W. Ahn, R. S. Ramakrishna. 2002. A Genetic Algorithm for Shortest Path Routing Problem and the Sizing of Populations. IEEE TRANSACTIONS ON EVOLUTIONARY COMPUTATION, VOL. 6, NO. 6, DECEMBER 2002.

[17] M. R. Girgis, T. M. Mahmoud1, H. F. Abd el- Hammed, and Z. M. El-Saghier. 2013. Routing And Capacity Assignment Problem in Computer Networks Using Genetic Algorithm. Information, Science Letters. Let 2, No. 1, 13-25 (2013).
[18] Mehta, A. Sharma. 2013. Observing the Effect of Elitism on the Performance of GA. International Journal of Advanced Research in Computer Science and Software Engineering. Volume 3, Issue 6, June 2013 Research Paper Available online at: www.ijarcsse.com.

[19] N. M. Razali, J.Geraghty. 2011. Genetic Algorithm Performance with Different Selection Strategies in Solving TSP. Proceedings of the World Congress on Engineering 2011 Vol II ,WCE 2011, July 6 - 8, 2011, London, U.K.

[20] M. Mitchell. 1999. An introduction to Genetic algorithm. $1^{\text {st }}$ edition, MIT Press, London, England.

[21] E.G. 1989. Genetic Algorithms in Search, Optimization and Machine Learning, University of Alabama. 